\title{
THE REDSHIFT DETERMINATION OF GRB 990506 AND GRB 000418 WITH THE ECHELLETE SPECTROGRAPH IMAGER ON KECK
}

\author{
J. S. Bloom, ${ }^{1,2,3}$ E. Berger, ${ }^{1}$ S. R. Kulkarni, ${ }^{1}$ S. G. Djorgovski, ${ }^{1}$ and D. A. Frail ${ }^{4}$ \\ Received 2002 November 20; accepted 2002 December 4
}

\begin{abstract}
Using the Echellete Spectrograph Imager (ESI) on the Keck II $10 \mathrm{~m}$ telescope we have measured the redshifts of the host galaxies of gamma-ray bursts GRB 990506 and GRB 000418, $z=1.30658 \pm 0.00004$ and $1.1181 \pm 0.0001$, respectively. Thanks to the excellent spectral resolution of ESI $(\lambda / \Delta \lambda=13000)$, we resolved the [O II] $\lambda 3727$ doublet in both cases. The measured redshift of GRB 990506 is the highest known for a darkburst GRB, though entirely consistent with the notion that dark and non-dark bursts have a common progenitor origin. The relative strengths of the [O II], He I, [Ne III], and $\mathrm{H} \gamma$ emission lines suggest that the host of GRB 000418 is a starburst galaxy, rather than a LINER or Seyfert 2. Since the host of GRB 000418 has been detected at submillimeter wavelengths, these spectroscopic observations suggest that the submillimeter emission is due to star formation (as opposed to AGN) activity. The [O II]-derived unobscured star formation rates are 13 and $55 M_{\odot} \mathrm{yr}^{-1}$ for the hosts of GRB 990506 and GRB 000418, respectively. In contrast, the star formation rate of the host of GRB 000418 derived from submillimeter observations is 20 times larger.
\end{abstract}

Key words: cosmology: miscellaneous — cosmology: observations — gamma rays

\section{INTRODUCTION}

The determination of redshifts continues to play a crucial role in the understanding of the physics of gamma-ray bursts (GRBs). Without redshifts we are unable to translate observed fluences and angular burst-host offsets into physically meaningful quantities. That redshift is an integral part of the physical modeling of the afterglow is best demonstrated by the immense difficulty in modeling the afterglow of GRB 980329 (Yost et al. 2002), a GRB that enjoys an abundance of panchromatic afterglow observations, but unfortunately lacked a measurement of redshift.

Emission spectra of GRB hosts themselves are valuable, given the potential use of GRBs to infer the star formation history of the universe. Since $\gamma$-rays penetrate dust, a GRBdefined sample does not suffer from the well-known biases of optical/UV samples. In addition, the high-accuracy astrometric localizations afforded by radio observations (with, e.g., the Very Large Array [VLA]) and/or X-ray (Chandra) offer significant observational advantages over a submillimeter-defined sample. Indeed, one great hope for the utility of GRBs is in revealing the star formation history at extremely high redshifts (e.g., Bromm \& Loeb 2002), since the brilliance of the prompt emission allows these events to be seen to redshifts well beyond 10 (Lamb \& Reichart 2000).

GRB 990506 is a canonical "dark burst" ${ }^{\text {" with a radio- }}$ detected afterglow with no optical transient emission (Taylor et al. 2000). Since no transient optical emission was detected from the afterglow, an optical absorption-line red-

${ }^{1}$ Palomar Observatory, Mail Stop 105-24, California Institute of Technology, Pasadena, CA 91125; jbloom@cfa.harvard.edu.

${ }^{2}$ Harvard Society of Fellows, 78 Mount Auburn Street, Cambridge, MA 02138.

${ }^{3}$ Harvard-Smithsonian Center for Astrophysics, MC 20, 60 Garden Street, Cambridge, MA 02138.

${ }^{4}$ National Radio Astronomy Observatory, Socorro, NM 87801.

${ }^{5}$ GRB 970828 is another such dark burst, with a similar detection history as GRB 990506 (Djorgovski et al. 2001): later optical observations showed that the radio-localized host of GRB 970828 was a dusty galaxy at $z \sim 1$. shift of GRB 990506 was impossible to obtain. Taylor et al. (2000) discovered an apparent two-galaxy system in optical data from Keck consistent with the radio transient position. Subsequent imaging from the Hubble Space Telescope (HST) revealed the putative two-galaxy system to be morphologically distinct (Holland et al. 2000). Bloom et al. (2002) later refined the radio $\rightarrow H S T$ astrometry to show that the radio afterglow position is only consistent with the southwest component; this component is identified as the host of GRB 990506. However, given the poor astrometric tie between the radio and the optical positions, as noted by Bloom et al., the GRB 990506-galaxy association is one of the more tenuous identifications of a host galaxy.

GRB 000418 is famous for two reasons. First, it is still one of the best cases for collimated ejecta of a GRB explosion (Berger et al. 2001). More importantly, the host galaxy has been detected in the radio (using VLA) and the submillimeter bands (using the Submillimeter Common User Bolometer Array [SCUBA] on the James Clerk Maxwell Telescope; see Berger et al. 2002). If the submillimeter emission arises from reprocessing of stellar UV light by dust (as opposed to arising from AGN activity), then the inferred star formation rate is very high. If so, this galaxy can potentially provide information about the nature of these ultraluminous galaxies and their contribution to the global star formation rate.

Here we present the spectroscopic observations of the hosts of GRB 000418 and GRB 990506. The discussion of GRB 000418 here expands our preliminary announcement of the redshift of the host (Bloom et al. 2000a). ${ }^{6}$ In $\S 2$ we describe the observations and reductions that led to the redshift determinations (presented in $\S \S 3-4$ ). In $\S 5$ we discuss some of the implications of the redshift detections in the context of GRB host galaxies.

\section{OBSERVATIONS AND REDUCTIONS}

The observations of both GRB host galaxies were obtained in the echellete mode of the Echellete Spectro-

\footnotetext{
${ }^{6}$ GCN Circulars are available at http://gcn.gsfc.nasa.gov.
} 
graph Imager (ESI; Sheinis et al. 2002) on the Keck II 10 m telescope atop Mauna Kea, Hawaii. The detector is a $2 \mathrm{~K} \times 4 \mathrm{~K}$ pixel Lincoln Labs $\mathrm{CCD}$ with low read noise $\left(\mathrm{rms} \approx 2.6 e^{-}\right)$and small dark current $\left(2.1 e^{-}\right.$pixel $\left.^{-1} \mathrm{hr}^{-1}\right)$. The wavelength coverage is complete from $\sim 3900 \AA$ to $1 \mu \mathrm{m}$, spanning 10 echellete orders (orders 15 through 6 , increasing with wavelength). The (inverse) dispersion is nearly constant at $11.5 \mathrm{~km} \mathrm{~s}^{-1}$ pixel $^{-1}$. For all of our observations we used the 1 !" $0 \times 20$ " 0 slit.

The putative host galaxies were identified as the galaxies nearest the astrometric position of the GRB afterglows. Bloom et al. (2002) estimated the chance that the afterglows were unrelated to the presumed host at $P_{\mathrm{ch}}=0.044$ (GRB 990506) and $P_{\mathrm{ch}}=0.0004$ (GRB 000418). The brightness of these galaxies are $R=24.0 \pm 0.3 \mathrm{mag}$ (GRB 000418; Bloom et al. 2000b) and $R=24.3 \pm 0.3 \mathrm{mag}$ (GRB 990506; Taylor et al. 2000). Both the galaxies are compact (half-light radius $<0$ ".2) as measured by STIS/HST observations (Metzger et al. 2000; Holland et al. 2000; Bloom et al. 2002). A summary of the observations of both GRB hosts is presented in Table 1. For both sources we centered the slit on a bright nearby star and then offset the telescope to the host galaxy by a distance derived from our ground-based astrometric solution of the field. Exposures of the listed spectrophotometric standard stars from Oke (1990) were obtained immediately following the last GRB host exposure. All science and calibration data were taken near the parallactic angle.

The data were reduced and calibrated using the standard spectral reduction packages in IRAF, ${ }^{7}$ primarily the packages CCDRED, NOAO/ONEDSPEC, and ECHELLE. For completeness we outline the steps taken. The data were first debiased using the overscan region of the CCD, and then bad pixels were replaced using FIXPIX. A flat field was constructed from five dome flats taken at the beginning of the night and applied to the science data. Cosmic rays were identified by finding statistically significant high-valued pixels in the ratio of each science image with the median of the science images. The cosmic-ray pixels were then replaced using FIXPIX. Using IDENTIFY, REIDENTIFY, and FITCOORDS, we characterized the curvature of the 10 echellete orders using a trace of a bright star, which we stepped along the slit with a constant $2^{\prime \prime}$ offset. A plate scale in the spatial direction for each echellete order was also obtained using these spectra. Rectification (setting the dispersion and spatial axes to be orthogonal) of the science image was performed using the task TRANSFORM with flux conservation.

${ }^{7}$ IRAF is distributed by the National Optical Astronomy Observatory, which is operated by the Association of Universities for Research in Astronomy, Inc., under cooperative agreement with the National Science Foundation.
In each image the 10 orders were then cut into individual long-slit two-dimensional spectra. The background sky was removed from each spectra using BACKGROUND. Since a feature near $8600 \AA$ was readily identifiable (see below) in order $7(8006 \AA<\lambda<9377 \AA)$ of each exposure of GRB 990506, we co-added the background subtracted spectra in order 7 using IMCOMBINE by determining the offset in pixels along the spatial direction of this feature from exposure to exposure. Spectrograms in the other orders were combined by scaling the offsets determined in order 7, using the empirically determined spatial scales in each order. Similarly, for GRB 000418 we determined offsets using the emission feature at $7900 \AA$.

The continuum of the host of GRB 990506 is not detected in the blue (orders 15 to 9, corresponding to $3756 \AA$ $<\lambda<7297 \AA$ ) and marginally detected in the three remaining red orders. Wavelength calibration was performed in the red orders using the night-sky lines only. The density of these lines in the red is sufficiently high that arc line spectra were not required for wavelength calibration. In the seventh echellete order a good wavelength solution was obtained with a seventh-order Legendre polynomial, which resulted in an rms uncertainty of $0.16 \AA$. The small airto-vacuum and heliocentric corrections were made using DISPTRANS, RVCORRECT, and DOPCOR. The flux calibration, using the standard star observations, was performed using STANDARD, SENSFUNC, and CALIBRATE.

We produced a final co-added flux-calibrated spectrum of the host of GRB 000418 using the five 1200 s exposures (Table 1) and following the reduction procedure as described above for GRB 990506. We dereddened the 000418 host spectrum using the extinction measure of $E(B-V)=0.3296$ from Schlegel et al. (1998). Unlike the case for GRB 990506, since the night was not photometric, a zero-point adjustment was made using the calibrated and extinction corrected Gunn $i$ magnitude $(i=23.38 \pm 0.10$ mag) from Berger et al. (2001) at the time of the spectroscopic observations. Using the zero point from Fukugita et al. (1995), the continuum flux at the effective wavelength of the Gunn $i$ (for a spectrum with $f_{\nu} \propto \nu^{-2}$ ) should have been $f_{\nu}(7973 \AA)=2.12 \mu \mathrm{Jy}$, whereas the measured value is 1.10 $\mu \mathrm{Jy}$. We thus scaled the spectrum by a factor of 1.93 and estimate a zero-point error of $30 \%$.

\section{THE REDSHIFT OF GRB 990506}

We visually identified an apparent doublet emission feature near $8600 \AA$ in order 7 of each 900 s spectrum. The spatial location of this feature was used to co-add the dithered spectra, as described above. Unfortunately, this apparent doublet is separated by a sky line $(\mathrm{O}-\mathrm{H}$ 28597.4$)$. However, given that the sky line and the fringing at this wavelength are both relatively weak, we are confident that

TABLE 1

Log OF SPECTROSCOPIC ObSERVATIONS OF GRB 990506 ANd GRB 000418

\begin{tabular}{cccccc}
\hline \hline Name & Date(UT) & $\begin{array}{c}\text { Int. Time } \\
(\mathrm{s})\end{array}$ & $\begin{array}{c}\text { Air Mass } \\
\text { (effective) }\end{array}$ & Standard Star & Photometric? \\
\hline GRB 990506 ............ & 2000 Apr 4.395 & $7 \times 900$ & 1.52 & Feige 67 & Yes \\
GRB 000418 …........ & 2000 May 1.319 & $1 \times 1200$ & 1.01 & Feige 34 & No \\
& 2000 May 2.403 & $5 \times 1200$ & 1.2 & Feige 66 & No \\
\hline
\end{tabular}


the sky subtraction did not significantly alter the morphology of the feature; that is, we believe the feature is indeed a doublet (such as [O II]) and not a single broad emission line (such as $\operatorname{Ly} \alpha$ ). In fact, the sky line only overlaps the redward wing of the bluer emission line; the shape and flux in the red emission line are largely unaffected by the sky line. A portion of the two-dimensional spectrogram of the host of GRB 990506 is displayed in Figure 1. The [O II] emission doublet is clearly visible, as is the faint continuum.

Using the $\chi^{2}$ minimization deblending algorithm in IRAF/SPLOT we preliminarily fitted two Gaussian profiles plus a continuum to the emission features in the surrounding region, allowing all the parameters to be fitted independently. The central wavelengths of the two emission lines were found to be $\lambda_{1}=8596.80 \AA$ and $\lambda_{2}=8603.25 \AA$. If we associate these lines with [O II] $\lambda \lambda 3727.092,3729.875$, then the implied redshift is $z_{1}=1.30657$ and $z_{2}=1.30658$. That these two redshifts agree, greatly strengthens the association of these lines with [O II] emission.

Given the interpretation of the emission features as due to $[\mathrm{O} \mathrm{II}]$, we refine the redshift determination by fixing the relative separation of the two features to be the ratio of the [O II] doublet in vacuo. Since the blueward line was closer in wavelength to the sky line, we also fix the Gaussian FWHM of the blueward feature to be that of the redward feature (3.9 pixels). The emission redshift of the host of GRB 990506 so determined is $z(990506)=1.306576 \pm 0.000042$. This includes the statistical uncertainty in the wavelength solution and the wavelength centering of the doublet.
A confirmation of the redshift using other emission lines proves difficult, as most of the other strong nebular lineemission features associated with star formation $(\mathrm{H} \alpha$ $\lambda 6563, \mathrm{H} \beta \lambda 4861$, [O III] $\lambda \lambda 4363,4959,5007)$ are redshifted outside the ESI bandpass. However, the detection of the galaxy in the $R$ band (Taylor et al. 2000) implies that the feature could certainly not be $\operatorname{Ly} \alpha \lambda 1216$.

Assuming a flat CDM cosmology (e.g., de Bernardis et al. 2000) with $H_{0}=65 \mathrm{~km} \mathrm{~s}^{-1} \mathrm{Mpc}^{-1}, \Omega_{M}=0.3$, and $\Lambda_{0}=0.7$ the luminosity distance to the galaxy is $3.056 \times 10^{28} \mathrm{~cm}$ and $1^{\prime \prime}$ is $9.03 \mathrm{kpc}$ (physical) in projection.

\section{THE REDSHIFT OF GRB 000418}

We detect a strong doublet at $\lambda \approx 7898 \AA$ and identify this doublet with the $[\mathrm{O}$ II] doublet due to emission from the underlying host galaxy at redshift $z \simeq 1.1181$. A subsequent search for associated star formation emission lines reveals the presence of three faint lines ( $\mathrm{He}$ I, [Ne III], $\mathrm{H} \gamma$ ) in the spectrum that corroborate the redshift. The detection of $\mathrm{H} \gamma$ is marginal relative to the other detections. The identifications of the lines are listed in Table 2. The spectrum in the vicinity of $[\mathrm{O}$ II] and $\mathrm{He}$ I and $\mathrm{Ne}$ III are shown in Figure 2. Unlike in the spectrum of GRB 990506, the [O II] doublet is not particularly affected/overshadowed by a neighboring sky line, although the proximity of a faint sky line just blueward of the 3727.11 A line adds to the uncertainty in the measured equivalent width (EW) and luminosity.

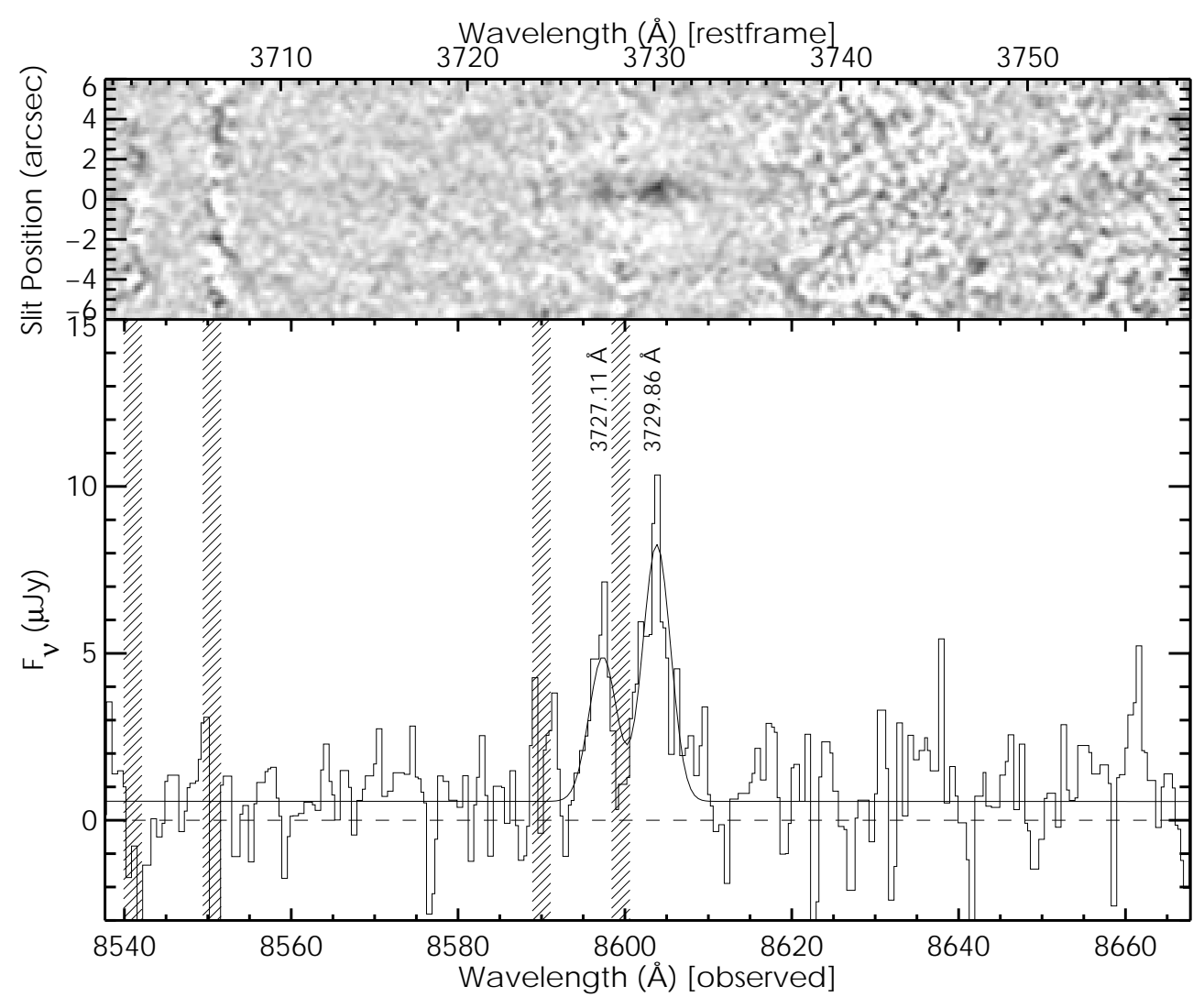

FIG. 1. - Summed spectrum of the host galaxy of GRB 990506 in the vicinity of the [O II] emission doublet. Top: Background subtracted two-dimensional spectrogram smoothed by a Gaussian with $\sigma=0.5 \AA$. The doublet is clearly visible at the center of the spectrogram and the faint continuum is also present. Bottom: One-dimensional spectrum. The solid line represents the fit of the continuum plus [O II] doublet flux to the data with the rest-frame wavelengths of the two emission lines noted. The hatched vertical lines mark the location of strong night-sky lines. 
TABLE 2

Emission-Line Properties of the Host of GRB 000418

\begin{tabular}{|c|c|c|c|c|c|}
\hline \multirow[b]{2}{*}{ LINE ID } & \multicolumn{2}{|c|}{ WAVELENGTH } & \multirow{2}{*}{$\begin{array}{c}F\left(\nu_{0}\right) \\
\left(\operatorname{ergs~} \mathrm{cm}^{2} \mathrm{~s}^{-1}\right)\end{array}$} & \multirow{2}{*}{$\begin{array}{l}\text { EW } \\
(\AA)\end{array}$} & \multirow[b]{2}{*}{$z$} \\
\hline & Rest Frame $(\AA)$ & Observed (Å) & & & \\
\hline \multirow[t]{2}{*}[\mathrm{O}\text{II}]{$\ldots \ldots \ldots \ldots \ldots \ldots$} & 3727.11 & 7894.42 & $2.8 \times 10^{-17}$ & $52 \pm 4$ & 1.11811 \\
\hline & 3729.86 & 7900.23 & $3.8 \times 10^{-17}$ & $69 \pm 5$ & 1.11810 \\
\hline$[\mathrm{Ne}$ III $] \ldots \ldots \ldots \ldots \ldots$ & 3869.85 & 8196.96 & $2 \times 10^{-18}$ & $\sim 7$ & 1.11816 \\
\hline He I ................. & 3888.65 & 8239.6 & $2 \times 10^{-18}$ & $\sim 20$ & 1.1189 \\
\hline $\mathrm{H} \gamma \ldots \ldots \ldots \ldots \ldots \ldots$ & 4341.69 & 9202.8 & $4 \times 10^{-18}$ & $\sim 20$ & 1.1196 \\
\hline
\end{tabular}

The luminosity-weighted redshift using the [O II], He I, and [Ne III] lines is $z(000418)=1.1181 \pm 0.0001$. Using the cosmology assumed above, the luminosity distance to the galaxy is $2.520 \times 10^{28} \mathrm{~cm}$ and $1^{\prime \prime}$ is $8.83 \mathrm{kpc}$ (physical) in projection.

\section{THE NATURE OF THE HOST GALAXIES}

\subsection{The Host Galaxy of GRB 990506}

We estimate the unobscured star formation in the host by measuring the flux of the [O II] line. First, we assess the accuracy of our flux calibration. At $8600 \AA$ we find that the flux of the continuum is $0.57 \mu \mathrm{Jy}$ with a red continuum, the slope of which is difficult to fit over a large wavelength range. Taking the continuum spectral index to be $0.2 \lesssim \alpha \lesssim 0.8$, with $f_{\nu} \propto \lambda^{\alpha}$, the flux at the central wavelength of the $R_{\mathrm{C}}$ filter is $0.54 \gtrsim f_{\nu}(6415 \AA) \gtrsim 0.45 \mu \mathrm{Jy}$. Using the zero point from Fukugita et al. (1995), the corresponding $R$-band spectroscopic magnitude is $24.37 \mathrm{mag} \lesssim R_{\mathrm{C}}$ (spect) $\lesssim 24.57 \mathrm{mag}$. This spectrophotometric flux estimate agrees reasonably well with the photometric measurement of $R=24.3 \pm 0.3$ mag from Taylor et al. (2000). From the difference $(\approx 0.1$ mag) we estimate a conservative systematic uncertainty of our flux calibration at $20 \%$.

Using the line-deblending method described above, we found the observed flux in the emission doublet to be $F_{8596}=(7.01 \pm 1.2) \times 10^{-18}$ ergs $\mathrm{s}^{-1} \mathrm{~cm}^{-2}$ and $F_{8603}=$ $(12.47 \pm 1.1) \times 10^{-18} \mathrm{ergs} \mathrm{s}^{-1} \mathrm{~cm}^{-2}$. According to the dust maps of Schlegel et al. (1998), the Galactic extinction in the direction of GRB 990506 is $E(B-V)=0.0686$ mag. Assuming the $R_{V}=3.1$ extinction curve of Cardelli et al. (1989) the dereddened flux at $8600 \AA$ is $10.7 \%$ higher than observed. The total dereddened flux in the $[\mathrm{O}$ II] line is thus $F_{[\mathrm{OII}}=(2.16 \pm 0.18) \times 10^{-17} \mathrm{ergs} \mathrm{s}^{-1} \mathrm{~cm}^{-2}$.

The total luminosity in the $\left[\begin{array}{ll}\mathrm{O} & \mathrm{II}\end{array}\right]$ line is $\left.L_{[\mathrm{O}} \mathrm{II}\right]=$ $(2.53 \pm 0.21) \times 10^{41} \mathrm{ergs} \mathrm{s}^{-1}$. We have no a priori determination of the intrinsic extinction due to dust external to the Galaxy, so this luminosity should be considered a lower limit. The implied unobscured star formation rate in the GRB host galaxy is, using the relation from Kennicutt (1992), $12.6 M_{\odot} \mathrm{yr}^{-1}$. This estimate is uncertain by $\sim 10 \%$ (statistical) and 20\% (systematic).

In the rest frame the total equivalent width is $\mathrm{EW}_{[\mathrm{O}}$ II] $=$ $36.5 \pm 5.44 \AA$ A. No comparison sample of field galaxy [O II] EW measures at comparable redshifts has yet been published, but it is clear that the EW is not especially higher than an extrapolation from the low-redshift sample of Hogg et al. (1998). (Aside from the redshift differences, we caution against the use of the Hogg sample as a representative sample for comparison to GRB hosts; galaxies in that sample were selected on the basis of optical magnitudes and, given the trend of decreasing EW with increasing apparent galaxy brightness, that sample systematically selects against galaxies of high EW.) Nevertheless, to this end, the star formation rate per unit mass does not appear to be particularly high for star-forming galaxies at comparable redshifts.

The observed EW in the $\left[\begin{array}{ll}\mathrm{OI}\end{array}\right]$ doublet lines are $\mathrm{EW}_{3727.11}=30.6 \pm 7.9 \AA$ and $\mathrm{EW}_{3729.86}=53.6 \pm 9.7 \AA$. The ratio of the lines $\mathrm{EW}_{3729} / \mathrm{EW}_{3727}=1.75 \pm 0.55$. This is consistent with the ratio expected (1.5) in the limit of low density in the host galaxy H II regions (Osterbrock 1974). Note that the formal error on this ratio, found assuming the statistical errors quoted above on both lines, is likely an overestimate of the true uncertainty since the uncertainty in the continuum level - which is essentially the same for both lines - dominates the statistical error.

\subsection{The Host Galaxy of GRB 000418}

The fluxes and equivalent widths of the observed lines are given in Table 2 and were found using the same deblending method as above. None of the lines appear to be extended beyond the instrumental resolution; that is, all of the lines are narrow. The total (dereddened) flux in the [O II] line is $F_{[\mathrm{OII}}=(1.4 \pm 0.2) \times 10^{-16} \mathrm{ergs} \mathrm{s}^{-1} \mathrm{~cm}^{-2}$ corresponding to a luminosity in the [O II] line of $L_{[\mathrm{O}}$ II] $=(1.1 \pm 0.16) \times 10^{42}$ $\mathrm{ergs} \mathrm{s}^{-1}$. The implied unobscured star formation rate in the GRB host galaxy is, using the relation from Kennicutt (1992), $55 M_{\odot} \mathrm{yr}^{-1}$. This estimate is uncertain by $\sim 10 \%$ (statistical) and 30\% (systematic).

The total observed equivalent width of the [O II] line is $\mathrm{EW}_{\text {obs }}=111 \pm 6 \AA$, corresponding to a rest-frame $\mathrm{EW}_{\text {rest }}=52.4 \pm 2.8 \AA$. This does appear to be higher than an extrapolation of the Hogg sample and is suggestive of high star formation per unit mass. The ratio of the [Ne III] to [O II] flux is of interest both as a starburst/AGN discriminator (e.g., Rola et al. 1997) and because several other GRB hosts appear to have a large ratio, indicating very hot $\mathrm{H}$ II regions (e.g., Bloom et al. 2001). The observed ratio, $\sim 0.03$, is lower than that inferred from GRB 970228 and rules out a Seyfert 2 origin of emission (following Fig. 4 of Rola et al. 1997). With the lines observed we cannot directly address whether the galaxy is a LINER (both $\mathrm{H} \beta$ and [O III] are redshifted outside the range of observability with ESI). However, assuming case $\mathrm{B}$ recombination and little intrinsic extinction, the implied $\mathrm{H} \beta$ flux is $F_{\mathrm{H} \beta} \approx F_{\mathrm{H} \gamma} / 0.474=$ $8.4 \times 10^{-18} \mathrm{ergs} \mathrm{s}^{-1} \mathrm{~cm}^{-2}$. The implied flux ratios of $[\mathrm{O} \mathrm{II}] /$ $\mathrm{H} \beta$ and $[\mathrm{Ne} \mathrm{III}] / \mathrm{H} \beta$, then, place this source comfortably near starburst galaxies and sufficiently away from the locus of known LINERs (Fig. $3 a$ of Rola et al. 1997). We thereby identify the host of GRB 000418 as a starburst galaxy. 

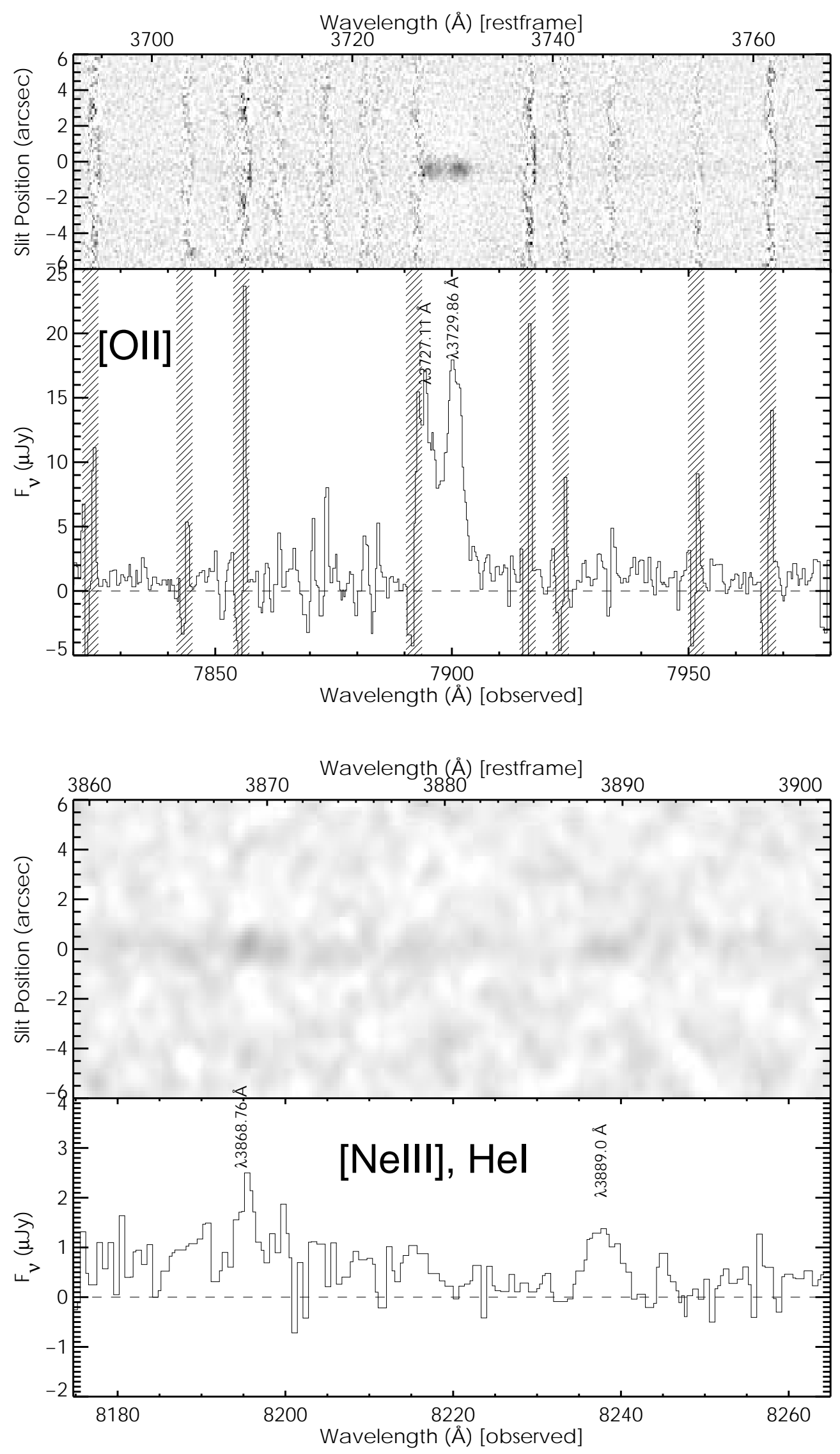

FIG. 2.-Summed spectrum of the host galaxy of GRB 000418 in the vicinity of (top) the [O II] emission doublet and (bottom) [Ne III] and He I. The background subtracted two-dimensional spectrogram is smoothed by a Gaussian with $\sigma=0.5 \AA$. As in Fig. 1 the doublet is clearly visible at the center of the spectrogram, and the continuum is also detected. The hatched vertical lines mark the location of strong night-sky lines. 
The radio and submillimeter detections of the host galaxy of GRB 000418 also indicate a high star formation rate. We estimated the star formation rate using the formulation of Yun \& Carilli (2002). As detailed in Berger et al. (2002), the flux density at $350 \mathrm{GHz}, F_{\nu}(350 \mathrm{GHz})=3.2 \mathrm{mJy}$, implies $\mathrm{SFR} \approx 700 M_{\odot} \mathrm{yr}^{-1}$, while the flux density at $8.46 \mathrm{GHz}$, $F_{\nu}(8.46 \mathrm{GHz})=51 \mu \mathrm{Jy}$, implies SFR $\approx 330 M_{\odot} \mathrm{yr}^{-1}$. For the radio-to-SFR conversion we assume $\alpha=0.6$ (Fomalont et al. 2002). The discrepancy between the two SFR estimates is expected, since in both cases there is a $\sim 25 \%$ uncertainty in the flux density. Thus, the SFR implied from the longwavelength observations is in the range $\sim 250-900 M_{\odot} \mathrm{yr}^{-1}$.

Clearly, this SFR estimate is about a factor of 10-20 higher than the estimate from the [O II] emission. This is not surprising, given that the [O II] luminosity is a measure of the unobscured SFR, and correction factors of 5-10 for high-redshift galaxies are not uncommon (Adelberger \& Steidel 2000). Interestingly, there was little to no host galaxy extinction inferred in the GRB afterglow itself (Berger et al. 2001).

\section{DISCUSSION AND CONCLUSION}

Traditionally, low-resolution spectroscopy $(R \approx 1000)$ has been used to determine emission-line redshifts of GRB hosts. Indeed, on the night previous to the $[\mathrm{O}$ II] detection of the host of GRB 990506 in echellete mode, a $1 \mathrm{hr}$ lowresolution long-slit prism spectrum was obtained and no lines were detected. This nondetection motivated our subsequent use of the spectrograph in echellete (mediumresolution) mode.

Medium- to high-resolution spectrometers have already been used for the detection of absorption-line redshifts of GRBs (Castro et al. 2000a, 2000b; Mirabal et al. 2002; Savaglio et al. 2002), but the redshifts of the hosts of GRB 000418 and GRB 990506 are the first emission-line redshifts found with a medium-resolution $(R=13000)$ echellete spectrograph.

This point is worth highlighting from an observational perspective. Given that the throughput of ESI in echellete mode $(\lesssim 12 \%)$ is approximately $40 \%$ smaller than that of the prism long-slit ("high-throughput") mode (see Sheinis et al. 2002), it is not immediately obvious why echellete spectroscopy would be able to detect the redshift of GRB 990506, whereas long-slit spectroscopy failed to detect the line. While both observing modes provide similar wavelength coverage and slit widths, however, the prism long-slit mode results in a lower dispersion with increasing wavelengths (from $1 \AA$ pixel $^{-1}$ at $4000 \AA$ to $\sim 10 \AA$ pixel $^{-1}$ at $10000 \AA$ ). This effect causes the numerous sky emission lines to overlap to the extent that, beyond about $6000 \AA$, almost all pixels are dominated by dispersed sky emission lines. In the echellete mode, the sky lines are always resolved, except for the largest slit widths. Even if sky subtraction were perfect, the noise from the high sky background levels in low-resolution mode more than mitigates, in terms of signal-to-noise ratio, against the gain in throughput.

Is it clear from previous studies that GRB hosts contain moderate levels of unobscured star formation, resulting in strong Balmer line, $[\mathrm{O}$ II], and Ly $\alpha$ emission-lines (see Djorgovski et al. 2001 for a review). Most GRBs, however, appear to occur at redshifts near unity, and so $\mathrm{H} \alpha$ and $\mathrm{Ly} \alpha$ lines are outside the range of detectability for optical CCDs. At such redshifts [O II], typically the third most luminous star-formation line, resides at $\lambda \gtrsim 6000 \AA(z \gtrsim 0.6)$ in the observer's frame; however, this is the onset of the wavelength regime where the prominent sky lines become numerous and densely packed. As a faint, typically narrow line (observed FWHM $\lesssim 6 \AA$ ), the [O II] line can be easily outshone from a nearby night-sky line when the dispersion is low. This was clearly the case in GRB 990506 (Fig. 1).

Medium-resolution spectroscopy also affords a better insight into the nature of any emission-line detections. Specifically, the ambiguity of single-line redshifts (see Stern et al. 2000) is essentially removed, since the resolution of ESI is large enough to resolve the [O II] doublet (rest-frame separation of $2.75 \AA$ ) for redshifts greater than $z \sim 0.5$.

From the optical lines we have shown that the unobscured star formation rates in the hosts of GRB 990506 and GRB 000418 are 13 and $55 M_{\odot} \mathrm{yr}^{-1}$, respectively. This may be contrasted with SFR $\sim 600 M_{\odot} \mathrm{yr}^{-1}$ from submillimeter and radio observations of GRB 000418 . Such a discrepancy has been observed in many high- $z$ galaxies, and it is probably due to dust obscuration. Such a trend, for GRB galaxies, has been previously noted by comparing infrared photometry with spectroscopic star formation indicators (Chary et al. 2002). More importantly, however, spectroscopy of GRB 000418 has allowed us to determine the redshift to a submillimeter galaxy. This is a significant result since there are only a handful of such galaxies with a measured redshift (e.g., Ledlow et al. 2002). This hints at the unique potential of GRB-selected galaxies in uncovering the redshift distribution of the population of submillimeter galaxies.

Since GRB 990506 is one of only a handful of well-studied dark bursts (also including GRB 000210, Piro et al. 2002; and GRB 970828, Djorgovski et al. 2001) the measurement of a redshift near the median of other long-duration bursts may suggest a common progenitor population. This emerging trend of similar redshift distributions will almost certainly be testable with the advent of systematic absorption redshift determinations for Swift bursts.

The authors thank the generous support of the staff of the W. M. Keck Foundation. J. S. B. gratefully acknowledges the fellowship from the Fannie and John Hertz Foundation and a research grant from the Harvard-Smithsonian Center for Astrophysics. S. R. K.'s research in GRBs is supported by the NSF and NASA. S. G. D. acknowledges partial funding from the Bressler Foundation. We thank S. Castro for helpful discussions concerning ESI reductions. The National Radio Astronomy Observatory is a facility of the NSF operated under cooperative agreement by Associated Universities, Inc. The authors wish to extend special thanks to those of Hawaiian ancestry on whose sacred mountain we are privileged to be guests. Without their generous hospitality, the observations presented herein would not have been possible. 


\section{REFERENCES}

Adelberger, K. L. \& Steidel, C. C. 2000, ApJ, 544, 218

Berger, E., Cowie, L. L., Kulkarni, S. R., Frail, D. A., Aussel, H., \& Barger, A. J. 2002, ApJ, submitted

Berger, E., et al. 2001, ApJ, 556, 556

Bloom, J. S., Diercks, A., Djorgovski, S. G., Kaplan, D., \& Kulkarni, S. R. 2000a, GCN Circ., 661, 1

Bloom, J. S., Diercks, A., Galama, T. J., Mahabal, A., Kulkarni, S. R., Harrison, F. A., Mao, P., \& Helfand, D. 2000b, GCN Circ., 689, 1

Bloom, J. S., Djorgovski, S. G., \& Kulkarni, S. R. 2001, ApJ, 554, 678

Bloom, J. S., Kulkarni, S. R., \& Djorgovski, S. G. 2002, AJ, 123, 1111

Bromm, V., \& Loeb, A. 2002, ApJ, 575, 111

Cardelli, J. A., Clayton, G. C., \& Mathis, J. S. 1989, ApJ, 345, 245

Castro, S. M., Diercks, A., Djorgovski, S. G., Kulkarni, S. R., Galama,

T. J., Bloom, J. S., Harrison, F. A., \& Frail, D. A. 2000a, GCN Circ., 605 Castro, S. M. et al. 2000b, GCN Circ., 851

Chary, R., Becklin, E. E., \& Armus, L. 2002, ApJ, 566, 229

de Bernardis, P., et al. 2000, Nature, 404, 955

Djorgovski, S. G., Frail, D. A., Kulkarni, S. R., Bloom, J. S., Odewahn, S. C., \& Diercks, A. 2001, ApJ, 562, 654

Djorgovski, S. G. et al. 2001, in Gamma-Ray Bursts in the Afterglow Era, ed. E. Costa, F. Frontera, \& J. Hjorth (New York: Springer), 218

Fomalont, E. B., Kellermann, K. I., Partridge, R. B., Windhorst, R. A., \& Richards, E. A. 2002, AJ, 123, 2402

Fukugita, M., Shimasaku, K., \& Ichikawa, T. 1995, PASP, 107, 945
Hogg, D. W., Cohen, J. G., Blandford, R., \& Pahre, M. A. 1998, ApJ, 504, 622

Holland, S., et al. 2000, GCN Circ., 731

Kennicutt, R. C. 1992, ApJ, 388, 310

Lamb, D. Q., \& Reichart, D. E. 2000, ApJ, 536, 1

Ledlow, M. J., Smail, I., Owen, F. N., Keel, W. C., Ivison, R. J., \& Morrison, G. E. 2002, ApJ, 577, L79

Metzger, M., et al. 2000, GCN Circ., 733

Mirabal, N., et al. 2002, ApJ, 578, 818

Oke, J. B. 1990, AJ, 99, 1621

Osterbrock, D. E. 1974, Astrophysics of Gaseous Nebulae (San Francisco: Freeman)

Piro, L., et al. 2002, ApJ, 577, 680

Rola, C. S., Terlevich, E., \& Terlevich, R. J. 1997, MNRAS, 289, 419

Savaglio, S., et al. 2002, GCN Circ., 1633

Schlegel, D. J., Finkbeiner, D. P., \& Davis, M. 1998, ApJ, 500, 525

Sheinis, A. I., Bolte, M., Epps, H. W., Kibrick, R. I., Miller, J. S., Radovan, M. V., Bigelow, B. C., \& Sutin, B. M. 2002, PASP, 114, 851

Stern, D., Bunker, A., Spinrad, H., \& Dey, A. 2000, ApJ, 537, 73

Taylor, G. B., Bloom, J. S., Frail, D. A., Kulkarni, S. R., Djorgovski, S. G., \& Jacoby, B. A. 2000, ApJ, 537, L17

Yost, S. A., et al. 2002, ApJ, 577, 155

Yun, M. S., \& Carilli, C. L. 2002, ApJ, 568, 88 